\title{
Modelo de Proceso Software Aplicado a la Revisión de la Accesibilidad WEB en Desarrollos Basados en IDE
}

\author{
Pedro A. Duarte, Sonia I. Mariño, Pedro L. Alfonzo, María V. Godoy \\ Facultad de Ciencias Exactas y Naturales y Agrimensura. \\ Universidad Nacional del Nordeste. \\ 9 de Julio 1449, 3400 Corrientes, Argentina. \\ 27.duarte.pedro@hotmail.com, simarinio@yahoo.com,plalfonzo@hotmail.com,mvgodoy@exa.unne.edu.ar
}

\begin{abstract}
Resumen- Se describe un modelo de proceso software incremental que incluye como aspecto relevante la Accesibilidad Web. Considerada ésta como un atributo de la calidad del software y un Requerimiento No funcional. Se expone el método propuesto, su validación en una solución tecnológica concreta y los resultados logrados. Para finalizar se esbozan algunas consideraciones a contemplar en el uso de los entornos de desarrollo integrado y la Accesibilidad Web.
\end{abstract}

Palabras clave - Modelo de proceso software, accesibilidad web, entornos de desarrollo integrado.

\section{INTRODUCTION}

La Accesibilidad Web (AW) implica el acceso universal a los contenidos dispuestos en la Web, independientemente del tipo de hardware, software, infraestructura de red, idioma, cultura, localización geográfica y capacidades de los usuarios [1]. Con esta idea nace la Iniciativa de Accesibilidad Web, conocida como WAI (Web Accessibility Initiative) [2].

Un sitio web con criterios de accesibilidad debe darle la posibilidad, a personas con discapacidad o no, de utilizarlo con independencia de las limitaciones que posean.

La web, desde sus principios se contempló que tendría como requisito el que el mismo fuese universal y accesible, sin embargo hoy en día existen problemas para muchas personas con discapacidades.

La Ingeniería del Software es la aplicación de un enfoque sistemático, disciplinado y cuantificable al desarrollo, operación, y mantenimiento del software [3]. Para [4] es el establecimiento y uso de principios de ingeniería robustos, orientados a obtener económicamente software que sea fiable y funcione eficientemente sobre máquinas reales.

Cuando se desarrollan proyectos informáticos se deben aplicar métodos y técnicas para resolver los problemas iniciales, esta disciplina aporta herramientas y procedimientos sobre los que se apoya la Ingeniería del Software (IS).

La IS mejora la calidad del producto software. Aumenta la productividad de los ingenieros del software facilitando el control de los procesos de desarrollo. Suministra a los integrantes de la construcción del software las bases para su desarrollo con calidad y eficiencia.

Según [3] el requerimiento de software es:

- Una condición o capacidad requerida por un usuario para resolver un problema o alcanzar un objetivo.

- Una condición o capacidad que debe cumplir o poseer un sistema o componente de sistema para satisfacer un contrato, estándar, especificación, o cualquier otro documento impuesto formalmente.

- Una representación documentada de una condición o capacidad de lo explicado en los puntos 1 о 2.

En el desarrollo de software se deben contemplar factores los relacionados con la calidad, tanto los funcionales como los no funcionales. Se define la calidad del software como "la concordancia con los requerimientos funcionales y de rendimiento explícitamente establecidos, con los estándares de desarrollo explícitamente documentados y con las características implícitas que se espera de todo software desarrollado profesionalmente" [5].

En el desarrollo de sistemas web, se carece de un enfoque que incluya determinados Requerimientos No Funcionales o RNF como la accesibilidad web desde etapas tempranas.

En [6], se presenta un estudio relacionado al retraso que sufre el tratamiento de los RNF en el desarrollo de aplicaciones Web y establece como base conceptual que se trata de requerimientos de calidad.

Como antecedente previo en [7], se abordó la evaluación de una aplicación web generada por un Entorno de Desarrollo Integrado (IDE) para la determinación de su accesibilidad. Un IDE es un entorno o marco de trabajo para la mayoría de los lenguajes de programación, contiene un editor de código, un compilador y un depurador, entre otros

Del estudio realizado en [7] y siguiendo a [8], se desprende que la especificación de requerimientos para el desarrollo de aplicaciones generadas a través de un IDE, se debe reforzar integrando estándares de calidad siendo uno de ellos la accesibilidad, a fin de obtener una descripción más completa y de mayor calidad en el producto, atendiendo a que Internet se consolidó en los últimos tiempos como la red global de información y dejó de tener un uso reservado para algunos actores de la población para pasar a ser de uso masivo.

\section{METODOLOGÍA}

\section{A. Método 1. Indagación en AW}

Etapa 1: Profundización del marco teórico, tomando como fuente documentos y herramientas proporcionados por [2], [9], [10], [11], [12], [13] y [14].

Etapa 2: Selección y estudio de herramientas disponibles en la web que verifican el cumplimiento de los principios y las pautas propuestas por la $\mathrm{W} 3 \mathrm{C}$, en tres niveles de conformidad A, AA y AAA.

Etapa 3: Sistematización y análisis de los datos. Los resultados obtenidos permitieron analizar el estado del arte de 
las herramientas disponibles, consideradas de importancia para determinar el nivel de accesibilidad de sitios web y elaborar posteriores estudios a partir de los resultados alcanzados.

\section{B. Método 2. Propuesta de modelo de proceso software con inclusión de la $A W$.}

Se diseñó y desarrollo de un software para instituciones educativas basado en un "modelo evolutivo incremental" propuesto por [15], dado que se ha optado por un entorno de desarrollo integrado (IDE) y considerando las Pautas de Accesibilidad al Contenido (WCAG 1.0) [16] propuesto por la $\mathrm{W} 3 \mathrm{C}$, las fases contemplaron:

- Incremento 1. Desarrollo de un Sistema Informático utilizando un IDE.

Etapa de comunicación: En esta etapa, se llevaron a cabo entrevistas en las cuales el entrevistado expresó sus requerimientos, las cuales se plasmaron en el sistema construido. Dentro de las cuales se encuentran:

- Requerimientos del usuario

- Requerimientos del sistema

- Requerimientos funcionales dentro de los cuales se encuentran los siguientes:

- Requerimientos no funcionales: Mejora del rendimiento de la velocidad de respuesta en un $90 \%$.

- Interfaz de usuario mejorado en un $90 \%$ para usuarios sin conocimientos.

- El software puede ser implementado en sistemas Windows y Linux.

- El sistema se implementará con Java.

- El sistema se entregará en un periodo no mayor a los 20 días hábiles de la solicitud.

- El sistema deberá cumplir con las Pautas de Accesibilidad para el Contenido Web (WCAG 1.0) [16].

- Requerimientos del dominio

Etapa de planeación: En esta etapa se realizó un plan para el desarrollo evolutivo incremental que se utilizó en la construcción del sistema. El plan de desarrollo consistió en la definición de las tareas que se realizarán y un calendario donde se proyectarán las actividades y los resultados durante el desarrollo del sistema. Este plan sirvió para cumplir los objetivos de la manera más adecuada y en los tiempos estipulados.

Algunas de las actividades vinculadas en el desarrollo del sistema son:

- Desarrollo del contenido.

- Ingeniería de requerimientos.

- Revisión de requisitos.

- Tareas de programación.

- Diseño de la interfaz.

- Diseño gráfico.

- Pruebas.

- Mantenimiento.

Etapa de modelado: Incluyo el análisis y el diseño del sistema, comprendiendo dos modelos: requerimientos $\mathrm{y}$ diseño. El primero, constituye los requerimientos del usuario destinatario del sistema, enfocados en diferentes ángulos, la información, las funciones y el comportamiento. Respecto al segundo, implico brindar detalles de la arquitectura, interfaz de usuario, nivel de componentes, y demás características del sistema que en el desarrollo de elaborar el producto con eficacia.
Esta etapa consistió en procesar y analizar los resultados que se obtuvieron en la etapa anterior, se definieron y organizaron los requerimientos; las cuales comprenden:

- Definición de servicios identificados para el sistema aportando la mayor parte de información.

- Definición de actores de estudio, es decir agrupaciones de actores definidos en la etapa de requerimientos.

- Modelos de clases de contenido, representado por un diagrama de clases basado en los requerimientos de almacenamiento de información.

- Modelo de secuencia para actores de estudio, realizando un secuencia de navegación para los diferentes tipos de actores que interactúen con el sistema

Etapa de construcción: Esta etapa consistió en un conjunto de tareas realizadas para la codificación del sistema en base al diseño previo, obteniendo un software finalizado para la entrega a los destinatarios.

Se debió hacer hincapié en la selección del lenguaje de programación a utilizar y las pruebas a realizar. Su correcta selección permitió una eficaz codificación; y posteriori implementar las pruebas de manera óptima, obteniendo un software libre de errores, para ser utilizado por parte de los destinatarios

Etapa de despliegue: Se incluyeron tres actividades: entrega, apoyo y retroalimentación. La mismas se realizaron varias veces hasta lograr una aproximación al producto final. En cada ciclo, con respecto a la entrega, se proporcionó un incremento operativo, que brindó un conjunto de funcionalidades utilizables. Respecto al apoyo, se entregaron documentaciones de ayudas a los usuarios relacionados a las funcionalidades y característicos hasta el momento incluidas en el sistema elaborado. Por último, la retroalimentación constituyó un proceso mediante el cual se incorporaron las modificaciones pertinentes a las funciones y características existentes, enfoque a implementar en el próximo incremento, si la institución educativa desea agregar funcionalidades o nuevos requerimientos.

Incremento 2. Se analizan las pautas WCAG 1.0 [16] y especifican los cambios a realizar.

Se diseñó e implementó un modelo de proceso para evaluar la AW, como lo indica la Fig. 1, se definieron fases iterativas: Diseño, Prototipo y Evaluación. La evaluación de AW debe ser constante a través de sus prototipos en etapa de desarrollo, para conseguir corregir errores de accesibilidad desde etapas tempranas del desarrollo. La evaluación al finalizar el desarrollo implica altos costos en las correcciones, es decir, es más económico corregir un pequeño error en éstas etapas, que rediseñar completamente el sistema informático.

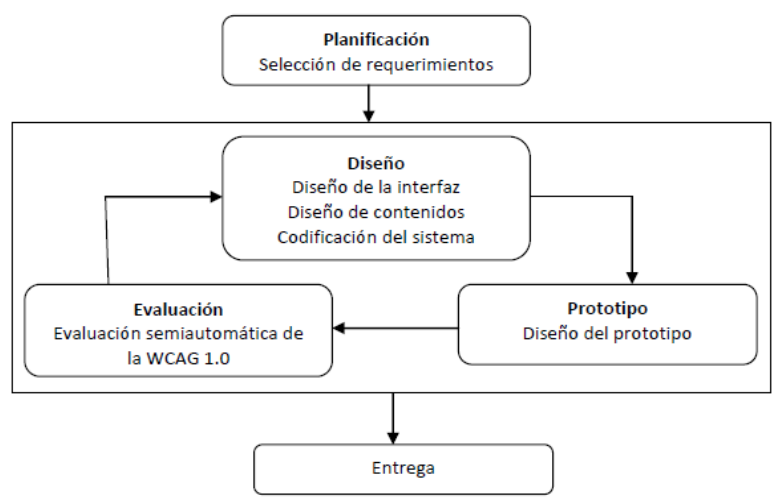

Fig. 1. Proceso de desarrollo (Fuente: elaboración propia)

Con la entrega de cada incremento y realizadas las evaluaciones correspondientes para evitar los problemas de 
AW, se continua el desarrollo de los módulos restantes. De este modo al realizar otro incremento en el cual se generen otros módulos, los anteriores desarrollados ya cumplirían con WCAG 1.0 [16].

\section{RESULTADOS}

A fin de validar la propuesta de modelo de proceso software con inclusión de la AW descripto en la sección 2, se exponen los resultados logrados al finalizar la realización de los dos incrementos.

\section{A. Incremento 1}

En [17], se describió un módulo del sistema informático educativo seleccionado para experimentar con el método propuesto. El sistema tiene un nivel de administración de los docentes, carreras, materias y alumnos, centralizando la información de manera local (Intranet) o a través de internet. Siendo un sistema informático web, puede ser utilizado bajo cualquier navegador de internet (Chrome, Internet Explorer, Firefox, entre otros).

\section{B. Incremento 2}

Con el propósito de detectar errores desde etapas tempranas de desarrollo del software, centrado en un requerimiento no funcional como la accesibilidad web, se describen los resultados derivados de la aplicación del proceso de desarrollo descripto anteriormente.

A pesar de que no todos los criterios de accesibilidad pueden ser evaluados de forma automática, los resultados obtenidos por estos métodos proporcionan indicadores que permiten un diagnóstico inicial del sitio Web analizado [8].

Para la evaluación semiautomática aplicada al sistema informático offline, se utilizó la herramienta TAW3 [18]. Además, se utilizó Chrome como navegador para la realización de las evaluaciones de AW.

TAW3 [18], permite analizar páginas individuales o sitios web completos, configurando el ámbito del análisis y los puntos de verificación a comprobar. Es decir,

- Ámbito del análisis: se indican los parámetros de exploración consistentes en tipología de enlaces (seguir enlaces dentro de un directorio o un dominio, seguir todos los enlaces o no seguir ninguno), niveles a analizar y número máximo de páginas.

- Configuración de pautas: permite seleccionar los puntos de verificación a comprobar en el análisis de las páginas. Se puede escoger un conjunto predefinido de puntos de verificación según los niveles de accesibilidad o crear un conjunto personalizado. También se permite la creación de comprobaciones personalizadas introduciendo una expresión regular o utilizando un asistente.

En la Tabla I se muestra una síntesis del análisis, donde se identifican el número de problemas encontrados durante el análisis de la AW aplicado cada una de las páginas, detectado automáticamente y aquellos que deben revisarse manualmente.

Las Tablas II y III detallan las pautas de prioridad 1 " $A$ " y "AA" detectadas automáticamente y deben corregirse.

En las Tablas IV, V, VI y VII se detallan las frecuencias de cada uno de los errores, evaluación realizada en todos los módulos del sistema, de forma "Automática" y "Manual" para los dos niveles de "Prioridad": A y AA.

De los errores encontrados se infiere la necesidad de abordar una evaluación en profundidad. Es decir, desde las interfaces de inicio hasta aquellas asociadas a la finalización de una determinada opción. Por ejemplo, desde el registro de un usuario hasta el pago de una cuota de un alumno determinado.
Por ello, para verificar la accesibilidad durante todo el proceso, se evaluaron las interfaces semiautomáticamente generadas por un IDE.

TABLA I. SíNTESIS DEL ANÁLISIS EFECTUADO CON TAW3.

\begin{tabular}{|c|c|c|c|}
\hline Módulos & Prioridad & $\begin{array}{c}\text { Revision } \\
\text { Automática }\end{array}$ & $\begin{array}{c}\text { Revision } \\
\text { Manual }\end{array}$ \\
\hline \multirow{2}{*}{ Login } & A & 1 & 42 \\
\hline & AA & 13 & 26 \\
\hline \multirow{2}{*}{ Home } & A & 1 & 73 \\
\hline & AA & 41 & 56 \\
\hline \multirow{2}{*}{ Cursos } & A & 21 & 167 \\
\hline & AA & 64 & 110 \\
\hline \multirow{2}{*}{$\begin{array}{l}\text { Instancias } \\
\text { de Cursos }\end{array}$} & A & 42 & 519 \\
\hline & AA & 224 & 166 \\
\hline \multirow{2}{*}{ Docentes } & A & 11 & 235 \\
\hline & AA & 102 & 111 \\
\hline \multirow{2}{*}{ Alumnos } & A & 21 & 371 \\
\hline & $\mathrm{AA}$ & 147 & 137 \\
\hline \multirow{2}{*}{ Pagos } & A & 22 & 334 \\
\hline & AA & 147 & 143 \\
\hline \multirow{2}{*}{$\begin{array}{l}\text { Inscribir } \\
\text { Alumnos }\end{array}$} & A & 2 & 273 \\
\hline & AA & 154 & 140 \\
\hline \multirow{2}{*}{$\begin{array}{l}\text { Registrar } \\
\text { Pagos }\end{array}$} & A & 1 & 249 \\
\hline & AA & 137 & 134 \\
\hline \multirow{2}{*}{ Facturas } & A & 21 & 297 \\
\hline & AA & 128 & 139 \\
\hline \multirow{2}{*}{ Usuarios } & A & 7 & 133 \\
\hline & AA & 66 & 90 \\
\hline \multirow{2}{*}{ Perfiles } & A & 7 & 110 \\
\hline & AA & 54 & 79 \\
\hline \multirow{2}{*}{ Informes } & $\mathrm{A}$ & 1 & 87 \\
\hline & AA & 44 & 65 \\
\hline
\end{tabular}

Los resultados demuestran como principales problemas detectados: Ausencia de etiquetas alternativas para identificar los elementos no textuales; la organización del documento de forma que pueda ser leído sin hoja de estilo y asegurarse que las páginas sigan siendo utilizables cuando se desconecten o no se soporte los script, applets y otros objetos programados. Lo expuesto implicaría el incumplimiento de algunos de los aspectos técnicos propuestos por la W3C, los cuales fueron corregidos posteriormente.

\section{CONCLUSIONES}

Numerosos sistemas informáticos se diseñan, desarrollan e implementan utilizando métodos y herramientas de la IS a fin de aplicar un enfoque disciplinado. Sin embargo, se ha detectado de revisiones bibliográficas que la "Accesibilidad Web" como un aspecto de la calidad del software y como un requerimiento no funcional, en general no se incorpora en el ciclo de vida de un sistema informático. 
TABLA II. DETALLE PAUTAS DE PRIORIDAD 1 "A".

\begin{tabular}{|c|}
\hline Prioridad 1: "A" \\
\hline 1.1 Proporcione un texto equivalente para todo elemento no textual. \\
\hline $\begin{array}{l}\text { 2.1 Asegúrese de que toda la información transmitida a través de los colores } \\
\text { también esté disponible sin color, por ejemplo mediante el contexto o por } \\
\text { marcadores. }\end{array}$ \\
\hline $\begin{array}{l}\text { 4.1 Identifique claramente los cambios en el idioma del texto del documento y } \\
\text { en cualquier texto equivalente (Por ejemplo, leyendas). }\end{array}$ \\
\hline 5.1 En las tablas de datos, identifique los encabezamientos de fila y columna. \\
\hline $\begin{array}{l}\text { 5.2 Para las tablas de datos que tienen dos o más niveles lógicos de } \\
\text { encabezamientos de fila o columna, utilice marcadores para asociarlas celdas } \\
\text { de encabezamiento y las celdas de datos. }\end{array}$ \\
\hline $\begin{array}{l}\text { 6.1 Organice el documento de forma que pueda ser leído sin hoja de estilo. } \\
\text { Por ejemplo, cuando un documento HTML es interpretado sin asociarlo a una } \\
\text { hoja de estilo, tiene que ser posible leerlo }\end{array}$ \\
\hline $\begin{array}{l}\text { 6.2 Asegúrese de que los equivalentes de un contenido dinámico son } \\
\text { actualizados cuando cambia el contenido dinámico. }\end{array}$ \\
\hline $\begin{array}{l}\text { 6.3 Asegure que las páginas sigan siendo utilizables cuando se desconecten o } \\
\text { no se soporten los scripts, applets u otros objetos programados. Si esto no es } \\
\text { posible, proporcione información equivalente en una página alternativa } \\
\text { accesible. }\end{array}$ \\
\hline $\begin{array}{l}\text { 7.1 Hasta que las aplicaciones de usuario permitan controlarlo, evite provocar } \\
\text { destellos en la pantalla. }\end{array}$ \\
\hline $\begin{array}{l}\text { 8.1 Haga los elementos de programación, tales como scripts y applets, } \\
\text { directamente accesibles o compatibles con las ayudas técnicas. }\end{array}$ \\
\hline $\begin{array}{l}11.4 \mathrm{Si} \text {, después de los mayores esfuerzos, no puede crear una página } \\
\text { accesible, proporcione un vínculo a una página alternativa que use tecnologías } \\
\mathrm{W} 3 \mathrm{C} \text {, sea accesible, tenga información (o funcionalidad) equivalente y sea } \\
\text { actualizada tan a menudo como la página (original) inaccesible. }\end{array}$ \\
\hline $\begin{array}{l}\text { 14.1 Utilice el lenguaje apropiado más claro y simple para el contenido de un } \\
\text { sitio. }\end{array}$ \\
\hline
\end{tabular}

TABLA III. DETALlE PAUTAS DE PRIORIDAD 2 "AA”.

\begin{abstract}
Prioridad 2 "AA"
2.2 Asegúrese de que las combinaciones de los colores de fondo y primer plano tengan el suficiente contraste para que sean percibidas por personas con deficiencias de percepción de color o en pantallas en blanco y negro.

3.1 Cuando exista un marcador apropiado, use marcadores en vez de imágenes para transmitir la información.

3.4 Utilice unidades relativas en lugar de absolutas al especificar los valores en los atributos de los marcadores de lenguaje y en los valores de las propiedades de las hojas de estilo.
\end{abstract}

3.5 Utilice elementos de encabezado para transmitir la estructura lógica y utilícelos de acuerdo con la especificación.

3.6 Marque correctamente las listas y los ítems de las listas.

3.7 Marque las citas. No utilice el marcador de citas para efectos de formato tales como sangrías.

5.3 No utilice tablas para maquetar, a menos que la tabla tenga sentido cuando se alinee. Por otro lado, si la tabla no tiene sentido, proporcione una alternativa equivalente (la cual debe ser una versión alineada).

5.4 Si se utiliza una tabla para maquetar, no utilice marcadores estructurales para realizar un efecto visual de formato.

6.4 Para los scripts y applets, asegúrese de que los manejadores de eventos sean independientes del dispositivo de entrada.

7.2 Hasta que las aplicaciones de usuario permitan controlarlo, evite el parpadeo del contenido (por ejemplo, cambio de presentación en periodos regulares, así como el encendido y apagado).

7.3 Hasta que las aplicaciones de usuario permitan congelar el movimiento de los contenidos, evite los movimientos en las páginas.

8.1 Haga los elementos de programación, tales como scripts y applets, directamente accesibles o compatibles con las ayudas técnicas.

9.3 Para los "scripts", especifique manejadores de evento lógicos mejor que manejadores de evento dependiente de dispositivos.

10.2 Hasta que las aplicaciones de usuario soporten explícitamente la asociación entre control de formulario y etiqueta, para todos los controles de formularios con etiquetas asociadas implícitamente, asegúrese de que la etiqueta está colocada adecuadamente.

11.1 Utilice tecnologías W3C cuando estén disponibles y sean apropiadas para la tarea y use las últimas versiones que sean soportadas.

11.2 Evite características desaconsejadas por las tecnologías W3C.

12.4 Asocie explícitamente las etiquetas con sus controles.

13.2 Proporcione metadatos para añadir información semántica a las páginas y sitios.

13.3 Proporcione información sobre la maquetación general de un sitio (por ejemplo, mapa del sitio o tabla de contenidos).

13.4 Utilice los mecanismos de navegación de forma coherente.
TABLA IV. RESUMEN ERRORES: ANÁlisis AutOMÁTICO: PRIORIDAD 1 "A".

\begin{tabular}{|l|c|c|c|c|c|c|c|c|c|c|c|c|}
\hline \multicolumn{10}{|c|}{ Análisis Automático: Prioridad 1 "A". } \\
\hline Criterios & $\mathbf{1 . 1}$ & $\mathbf{2 . 1}$ & $\mathbf{4 . 1}$ & $\mathbf{5 . 1}$ & $\mathbf{5 . 2}$ & $\mathbf{6 . 1}$ & $\mathbf{6 . 2}$ & $\mathbf{6 . 3}$ & $\mathbf{7 . 1}$ & $\mathbf{8 . 1}$ & $\mathbf{1 1 . 4}$ & $\mathbf{1 4 . 1}$ \\
\hline Login & 1 & 0 & 0 & 0 & 0 & 0 & 0 & 0 & 0 & 0 & 0 & 0 \\
\hline Home & 1 & 0 & 0 & 0 & 0 & 0 & 0 & 0 & 0 & 0 & 0 & 0 \\
\hline Cursos & 21 & 0 & 0 & 0 & 0 & 0 & 0 & 0 & 0 & 0 & 0 & 0 \\
\hline $\begin{array}{l}\text { Instancias } \\
\text { de Cursos }\end{array}$ & 41 & 0 & 0 & 0 & 0 & 0 & 0 & 1 & 0 & 0 & 0 & 0 \\
\hline Docentes & 11 & 0 & 0 & 0 & 0 & 0 & 0 & 0 & 0 & 0 & 0 & 0 \\
\hline Alumnos & 21 & 0 & 0 & 0 & 0 & 0 & 0 & 0 & 0 & 0 & 0 & 0 \\
\hline Pagos & 21 & 0 & 0 & 0 & 0 & 0 & 0 & 1 & 0 & 0 & 0 & 0 \\
\hline $\begin{array}{l}\text { Inscribir } \\
\text { Alumnos }\end{array}$ & 1 & 0 & 0 & 0 & 0 & 0 & 0 & 1 & 0 & 0 & 0 & 0 \\
\hline $\begin{array}{l}\text { Registrar } \\
\text { Pagos }\end{array}$ & 1 & 0 & 0 & 0 & 0 & 0 & 0 & 0 & 0 & 0 & 0 & 0 \\
\hline Facturas & 21 & 0 & 0 & 0 & 0 & 0 & 0 & 0 & 0 & 0 & 0 & 0 \\
\hline Usuarios & 7 & 0 & 0 & 0 & 0 & 0 & 0 & 0 & 0 & 0 & 0 & 0 \\
\hline Perfiles & 7 & 0 & 0 & 0 & 0 & 0 & 0 & 0 & 0 & 0 & 0 & 0 \\
\hline Informes & 1 & 0 & 0 & 0 & 0 & 0 & 0 & 0 & 0 & 0 & 0 & 0 \\
\hline
\end{tabular}

TABLA V. Resumen ERROREs: ANÁlisis MANUAL: PRIORIDAD 1 "A".

\begin{tabular}{|l|c|c|c|c|c|c|c|c|c|c|c|c|}
\hline \multicolumn{10}{|c|}{ Análisis Manual Prioridad 1 "A". } \\
\hline Criterios & $\mathbf{1 . 1}$ & $\mathbf{2 . 1}$ & $\mathbf{4 . 1}$ & $\mathbf{5 . 1}$ & $\mathbf{5 . 2}$ & $\mathbf{6 . 1}$ & $\mathbf{6 . 2}$ & $\mathbf{6 . 3}$ & $\mathbf{7 . 1}$ & $\mathbf{8 . 1}$ & $\mathbf{1 1 . 4}$ & $\mathbf{1 4 . 1}$ \\
\hline Login & 2 & 1 & 1 & 1 & 1 & 13 & 5 & 10 & 1 & 5 & 1 & 1 \\
\hline Home & 5 & 1 & 1 & 3 & 3 & 33 & 6 & 12 & 1 & 6 & 1 & 1 \\
\hline Cursos & 24 & 1 & 1 & 3 & 4 & 103 & 7 & 14 & 1 & 7 & 1 & 1 \\
\hline $\begin{array}{l}\text { Instancias } \\
\text { de Cursos }\end{array}$ & 45 & 1 & 1 & 3 & 4 & 422 & 10 & 20 & 1 & 10 & 1 & 1 \\
\hline Docentes & 14 & 1 & 1 & 3 & 4 & 169 & 10 & 20 & 1 & 10 & 1 & 1 \\
\hline Alumnos & 24 & 1 & 1 & 3 & 4 & 295 & 10 & 20 & 1 & 10 & 1 & 1 \\
\hline Pagos & 26 & 1 & 1 & 3 & 4 & 256 & 10 & 20 & 1 & 10 & 1 & 1 \\
\hline $\begin{array}{l}\text { Inscribir } \\
\text { Alumnos }\end{array}$ & 4 & 1 & 1 & 4 & 7 & 213 & 10 & 20 & 1 & 10 & 1 & 1 \\
\hline $\begin{array}{l}\text { Registrar } \\
\text { Pagos }\end{array}$ & 4 & 1 & 1 & 3 & 6 & 195 & 9 & 18 & 1 & 9 & 1 & 1 \\
\hline Facturas & 26 & 1 & 1 & 3 & 4 & 219 & 10 & 20 & 1 & 10 & 1 & 1 \\
\hline Usuarios & 10 & 1 & 1 & 3 & 4 & 83 & 7 & 14 & 1 & 7 & 1 & 1 \\
\hline Perfiles & 10 & 1 & 1 & 3 & 4 & 60 & 7 & 14 & 1 & 7 & 1 & 1 \\
\hline Informes & 4 & 1 & 1 & 5 & 5 & 44 & 6 & 12 & 1 & 6 & 1 & 1 \\
\hline
\end{tabular}

La aplicación del proceso de desarrollo propuesto para evaluar la accesibilidad a los contenidos Web y a través del estudio abordado ha permitido verificar que, aún cuando los IDE brindan alternativas para agilizar el prototipado y el desarrollo rápido, carecen de determinados aspectos de calidad, como por ejemplo incluir en sus plantillas el aseguramiento del cumplimiento de algunos criterios de la AW.

Por lo expuesto se sugiere incluir este aspecto de la IS desde etapas tempranas del modelo de proceso del software.

Se hipotetiza que numerosos problemas de accesibilidad localizados en los sitios podrían fundamentarse en el desconocimiento de los "Criterios de Accesibilidad" por parte de los desarrolladores. Por ello, aplicar y difundir estos criterios en el Sector Software y Servicios. Informáticos, se considera como un aporte a la comunidad. 
TABLA VI. RESUMEN ERRORES: ANÁLISIS AUTOMÁTICO: PRIORIDAD 2 "AA".

\begin{tabular}{|c|c|c|c|c|c|c|c|c|c|c|c|c|c|c|c|c|c|c|c|c|c|c|c|}
\hline \multicolumn{110}{|c|}{ Análisis Automático Prioridad 2 AA". } \\
\hline Criterios & $\mathbf{2 . 2}$ & $\mathbf{3 . 1}$ & $\mathbf{3 . 3}$ & $\mathbf{3 . 4}$ & $\mathbf{3 . 5}$ & $\mathbf{3 . 6}$ & $\mathbf{3 . 7}$ & $\mathbf{5 . 3}$ & $\mathbf{5 . 4}$ & $\mathbf{6 . 4}$ & $\mathbf{7 . 2}$ & $\mathbf{7 . 3}$ & $\mathbf{8 . 1}$ & $\mathbf{9 . 3}$ & $\mathbf{1 0 . 2}$ & $\mathbf{1 1 . 1}$ & $\mathbf{1 1 . 2}$ & $\mathbf{1 2 . 3}$ & $\mathbf{1 2 . 4}$ & $\mathbf{1 3 . 1}$ & $\mathbf{1 3 . 2}$ & $\mathbf{1 3 . 3}$ & $\mathbf{1 3 . 4}$ \\
\hline Login & 0 & 0 & 0 & 8 & 1 & 0 & 0 & 0 & 0 & 0 & 0 & 0 & 0 & 0 & 0 & 0 & 2 & 2 & 0 & 0 & 0 & 0 & 0 \\
\hline Home & 0 & 0 & 0 & 0 & 29 & 1 & 0 & 0 & 0 & 0 & 0 & 0 & 0 & 0 & 0 & 1 & 0 & 0 & 0 & 0 & 0 & 0 & 0 \\
\hline Cursos & 0 & 0 & 0 & 0 & 47 & 1 & 0 & 0 & 0 & 0 & 0 & 0 & 0 & 0 & 0 & 0 & 15 & 1 & 0 & 0 & 0 & 0 & 0 \\
\hline Instancias de Cursos & 0 & 0 & 0 & 0 & 189 & 1 & 0 & 0 & 0 & 0 & 0 & 0 & 0 & 0 & 0 & 0 & 31 & 0 & 0 & 0 & 0 & 0 & 0 \\
\hline Docentes & 0 & 0 & 0 & 74 & 1 & 1 & 0 & 0 & 0 & 0 & 0 & 0 & 0 & 0 & 0 & 0 & 24 & 0 & 3 & 0 & 0 & 0 & 0 \\
\hline Alumnos & 0 & 0 & 0 & 119 & 1 & 0 & 0 & 0 & 0 & 0 & 0 & 0 & 0 & 0 & 0 & 0 & 24 & 0 & 3 & 0 & 0 & 0 & 0 \\
\hline Pagos & 0 & 0 & 0 & 120 & 1 & 0 & 0 & 0 & 0 & 0 & 0 & 0 & 0 & 0 & 0 & 0 & 22 & 0 & 4 & 0 & 0 & 0 & 0 \\
\hline Inscribir Alumnos & 0 & 0 & 0 & 121 & 1 & 0 & 0 & 0 & 0 & 0 & 0 & 0 & 0 & 0 & 0 & 0 & 25 & 0 & 7 & 0 & 0 & 0 & 0 \\
\hline Registrar Pagos & 0 & 0 & 0 & 104 & 1 & 0 & 0 & 0 & 0 & 0 & 0 & 0 & 0 & 0 & 0 & 0 & 26 & 0 & 6 & 0 & 0 & 0 & 0 \\
\hline Facturas & 0 & 0 & 0 & 103 & 1 & 0 & 0 & 0 & 0 & 0 & 0 & 0 & 0 & 0 & 0 & 0 & 20 & 0 & 4 & 0 & 0 & 0 & 0 \\
\hline Usuarios & 0 & 0 & 0 & 45 & 1 & 0 & 0 & 0 & 0 & 0 & 0 & 0 & 0 & 0 & 0 & 0 & 19 & 0 & 1 & 0 & 0 & 0 & 0 \\
\hline Perfiles & 0 & 0 & 0 & 36 & 1 & 0 & 0 & 0 & 0 & 0 & 0 & 0 & 0 & 0 & 0 & 0 & 16 & 0 & 1 & 0 & 0 & 0 & 0 \\
\hline Informes & 0 & 0 & 0 & 28 & 1 & 0 & 0 & 0 & 0 & 0 & 0 & 0 & 0 & 0 & 0 & 0 & 15 & 0 & 0 & 0 & 0 & 0 & 0 \\
\hline
\end{tabular}

TABLA VII. RESUMEN ERRORES: ANÁLISIS MANUAL: PRIORIDAD 2 "AA".

\begin{tabular}{|c|c|c|c|c|c|c|c|c|c|c|c|c|c|c|c|c|c|c|c|c|c|c|c|}
\hline \multicolumn{24}{|c|}{ Análisis Manual Prioridad 2 "AA". } \\
\hline Criterios & 2.2 & 3.1 & 3.3 & 3.4 & 3.5 & 3.6 & 3.7 & 5.3 & 5.4 & 6.4 & 7.2 & 7.3 & 8.1 & 9.3 & 10.2 & 11.1 & 11.2 & 12.3 & 12.4 & 13.1 & 13.2 & 13.3 & 13.4 \\
\hline Login & 1 & 1 & 0 & 1 & 0 & 1 & 1 & 2 & 0 & 6 & 1 & 1 & 5 & 1 & 1 & 1 & 0 & 0 & 0 & 0 & 1 & 1 & 1 \\
\hline Home & 4 & 1 & 0 & 9 & 1 & 0 & 1 & 1 & 0 & 5 & 19 & 1 & 1 & 6 & 1 & 1 & 1 & 0 & 0 & 0 & 1 & 1 & 1 \\
\hline Cursos & 23 & 1 & 0 & 8 & 1 & 0 & 1 & 1 & 0 & 8 & 1 & 35 & 1 & 1 & 7 & 15 & 1 & 1 & 0 & 0 & 0 & 1 & 1 \\
\hline Instancias de Cursos & 44 & 1 & 0 & 8 & 2 & 1 & 1 & 1 & 0 & 8 & 1 & 52 & 1 & 1 & 10 & 29 & 1 & 1 & 0 & 0 & 0 & 1 & 1 \\
\hline Docentes & 13 & 1 & 8 & 2 & 0 & 1 & 1 & 8 & 1 & 40 & 1 & 1 & 10 & 17 & 1 & 1 & 0 & 1 & 0 & 1 & 1 & 1 & 1 \\
\hline Alumnos & 23 & 1 & 8 & 2 & 0 & 1 & 1 & 8 & 1 & 47 & 1 & 1 & 10 & 24 & 1 & 1 & 0 & 1 & 0 & 3 & 1 & 1 & 1 \\
\hline Pagos & 25 & 1 & 8 & 2 & 0 & 1 & 1 & 8 & 1 & 45 & 1 & 1 & 10 & 22 & 1 & 1 & 0 & 1 & 0 & 11 & 1 & 1 & 1 \\
\hline Inscribir Alumnos & 3 & 1 & 8 & 2 & 0 & 1 & 1 & 11 & 3 & 58 & 1 & 1 & 10 & 33 & 1 & 1 & 0 & 1 & 0 & 1 & 1 & 1 & 1 \\
\hline Registrar Pagos & 3 & 1 & 8 & 2 & 0 & 1 & 1 & 9 & 3 & 56 & 1 & 1 & 9 & 33 & 1 & 1 & 0 & 1 & 0 & 1 & 1 & 1 & 1 \\
\hline Facturas & 25 & 1 & 8 & 2 & 0 & 1 & 1 & 8 & 1 & 43 & 1 & 1 & 10 & 20 & 1 & 1 & 0 & 1 & 0 & 11 & 1 & 1 & 1 \\
\hline Usuarios & 9 & 1 & 8 & 1 & 0 & 1 & 1 & 8 & 1 & 30 & 1 & 1 & 7 & 10 & 1 & 1 & 0 & 1 & 0 & 5 & 1 & 1 & 1 \\
\hline Perfiles & 9 & 1 & 8 & 1 & 0 & 1 & 1 & 7 & 1 & 27 & 1 & 1 & 7 & 7 & 1 & 1 & 0 & 1 & 0 & 1 & 1 & 1 & 1 \\
\hline Informes & 3 & 1 & 12 & 1 & 0 & 1 & 1 & 8 & 23 & 1 & 1 & 6 & 1 & 1 & 1 & 0 & 1 & 1 & 1 & 1 & 1 & 1 & 1 \\
\hline
\end{tabular}

\section{REFERENCIAS}

[1] Guía Breve de Accesibilidad Web. [En línea]. Disponible en: http://www.w3c.es/Divulgacion/GuiasBreves/Accesibilidad. [Accedido: 2-mar-2015].

[2] Web Accessibility Initiative. [En línea]. Disponible en: http://www.w3c.es/traducciones/es/wai/intro/accessibility [Accedido: 5-mar-2015].

[3] IEEE 610.12-1990. IEEE Standard Glossary of Software Engineering Terminology.

[4] F. L. Bauer, "Software Engineering", Information Processing 71, 1972.

[5] I. Sommerville, "Ingeniería del Software".7 $7^{\mathrm{a}}$ Edición. Ed. Pearson, 2005.

[6] S. Del Valle Rojo y A. Oliveros, "Requerimientos no funcionales para aplicaciones Web", 13th. Argentine Symposium on Software Engineering, ASSE 2012. [En línea]. Disponible en: www.4ljaiio.org.ar /sites/ default/files/432_ASSE_2012.pdf. [Accedido: 2-mar-2015].

[7] P. Duarte, S. I. Mariño, P. L. Alfonzo y M. V. Godoy, "Evaluación de la accesibilidad en software generado por un entorno de desarrollo integrado", Técnica Administrativa, Vol. 14, no 1, 2015.

[8] S. Mariño, M. Godoy, P. Alfonzo, J. Acevedo, L. Gómez Solís y A. Fernández Vázquez, "Accesibilidad en la definición de requerimientos no funcionales. Revisión de herramientas", Multiciencias, vol 12, no 3, pp. 305-312, 2012.

[9] CTIC. Centro tecnológico. La familia TAW. [En línea]. Disponible http://www.tawdis.net/tools/familiaTaw.html?lang=es. [Accedido: 2-mar-2015].
[10] J. Díaz Bossini y L. Moreno, "Accessibility to mobile interfaces for older people". 5th International Conference on Software Development and Technologies for Enhancing Accessibility and Fighting Info-exclusion. DSAI 2013, Procedia Computer Science 27, 2014.

[11] A. E. Martín, G. Gaetán, V. E. Saldaño, G. Miranda, S. Pastrana, D. Vilte y E. Gómez Vega, "Técnicas y herramientas para desarrollo de sitios web accesibles", In XV Workshop de Investigadores en Ciencias de la Computación. Argentina, 2013.

[12] C. De Oleo Moreta y L. Rodríguez Baena, "Pautas, métodos y herramientas de evaluación de accesibilidad web". Ventana Informática, no 28, enero-junio, 2013.

[13] J. J. Acevedo, L. Gómez Solís, S. I. Mariño y M. V. Godoy, “A guidelines for evaluating web accessibility, Level A", Journal of Computer Science \& Technology. JCS\&T. Vol. 13, no. 2. pp. 76-83, 2013.

[14] M. L. Sánchez Gordón y L. Moreno, "Toward an integration of Web accessibility into testing processes". 5th International Conference on Software Development and Technologies for Enhancing, Accessibility and Fighting Info-exclusion, DSAI 2013. Procedia Computer Science. Vol. 27. pp. 281 - 291, 2014.

[15] R. Pressman, "Ingeniería del software. Un enfoque práctico". $7^{\mathrm{a}}$ Edición. Mexico: Ed. Mc Graw-Hill Interamericana, 2010.

[16] WCAG 1.0, Web Content Accessibility. Guidelines (WCAG) 2.0. [En línea]. Disponible en: http://www.w3.org/TR/ WCAG10/. [Accedido: 6-mar-2015].

[17] P. Duarte, S. I. Mariño y P. L. Alfonzo, "Una solución para gestionar los pagos en instituciones educativas". Pistas Educativas, no. 110, Agosto-Diciembre 2014.

[18] TAW3. Versión descargable de TAW3 (WCAG 1.0). [En línea]. Disponible en: http://www.tawdis.net/tools/accesibilidad/desk top/?lang=es\# [Accedido: 2-mar-2015]. 


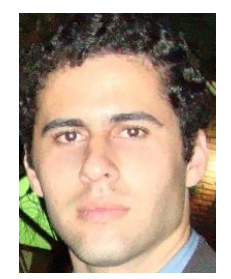

Pedro Duarte. Lic. en Sistemas de Información. Graduado de la Facultad de Ciencias Exactas y Naturales y Agrimensura - Ex Becario de la Facultad de Ciencias Exactas y Naturales y Agrimensura. Universidad Nacional del Nordeste (UNNE), Desarrollador software.

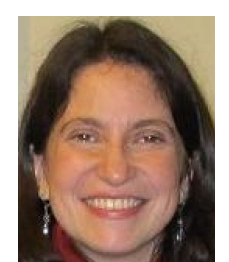

Sonia I. Mariño. Docente-Investigadora, Profesora Titular, Dedicación Exclusiva, del Departamento de Informática de la Facultad de Ciencias Exactas de la Universidad Nacional del Nordeste. Licenciada en Sistemas. Es Magíster en Informática y Computación. (UNNE - Universidad de Cantabria - España)., Magíster en Epistemología y Metodología de la Investigación Científica (Facultad de Humanidades - UNNE). Cursa el Doctorado en "Ciencias Cognitivas", Facultad de Humanidades (UNNE).

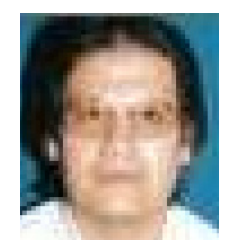

Pedro L. Alfonzo. Docente-Investigador. Experto en Estadística y Computación (Facultad de Ciencias Exactas y Naturales y Agrimensura UNNE). Especialista en Ingeniería de Software (Universidad Nacional de la Plata). Magíster en Ingeniería de Software (Universidad Nacional de La Plata).

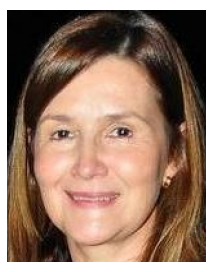

María V. Godoy. Docente-Investigadora, Profesora Titular, Dedicación Exclusiva, del Departamento de Informática de la Facultad de Ciencias Exactas de la Universidad Nacional del Nordeste. Experta en Estadística y Computación, y Licenciada en Sistemas. Es Magíster en Informática y Computación. (UNNE - Universidad de Cantabria - España). Cursa el Doctorado en "Ciencias Cognitivas", Facultad de Humanidades (UNNE). 